\title{
An association study between USP34 and polycystic ovary syndrome
}

\author{
Shigang Zhao 1,2,3,4,5t, Ye Tian ${ }^{1 \dagger}$, Wei Zhang ${ }^{6 \dagger}$, Xiuye Xing ${ }^{2,3,4,5}$, Tao Li ${ }^{2,3,4,5}$, Hongbin Liü ${ }^{2,3,4,5}$, Tao Huang ${ }^{2,3,4,5}$, \\ Yunna Ning ${ }^{2,3,4,5}$, Han Zhao ${ }^{2,3,4,5^{*}}$ and Zi-Jiang Chen ${ }^{1,2,3,4,5}$
}

\begin{abstract}
Background: Polycystic ovary syndrome (PCOS) is a complex multifactor disorder and genetic factors have been implicated in its pathogenesis. Our previous genome-wide association study (GWAS) had identified allele frequencies in several single nucleotide polymorphisms (SNPs) in gene USP34 (Ubiquitin-Specific Protease 34) were significantly different between PCOS cases and controls. This study was aimed to replicate the previous results in another independent cohort.
\end{abstract}

Methods: One thousand two hundred eighteen PCOS cases and 1057 controls were recruited. Genotyping of two SNPs (rs17008097 and rs17008940) in USP34 gene were performed by TaqMan-MGB probe assay and genotype-phenotype analysis was conducted subsequently.

Results: The differences of allele or genotype frequencies were not significant statistically between PCOS and controls, even after age and BMI adjustment. For clinical and metabolic features (LH, T and HOMA-IR) analysis in PCOS cases, no statistical differences among three genotypes of rs 17008097 and rs 17008940 were found. However, rs 17008940 was shown to be slightly associated with BMI in PCOS cases rather than in controls, even after age adjustment (TC vs CC $P=0.006, O R=1.042,95 \%$ Cl 1.012-1.073; TT vs CC $P=0.037, O R=1.050,95 \% C l 1.003-1.100$ ).

Conclusions: USP34 gene polymorphisms (rs17008097 and rs17008940) may not be associated with PCOS in the Han Chinese women.

Keywords: Polycystic ovary syndrome, USP34, SNPs, Association

\section{Background}

Polycystic ovary syndrome (PCOS) is a kind of reproductive and metabolic disorder characterized by hyper-androgen and insulin resistance, which affects $6-8 \%$ of reproductiveaged women in Caucasian and $5.6 \%$ in Chinese [1-3]. Clinical diagnosis of PCOS is made on the basis of at least two following criteria after excluding other related diseases: oligo- or anovulation, clinical or biochemical hyperandrogenism and polycystic ovaries under ultrasound [4]. The etiology of PCOS is not well understood yet. However, it's now widely accepted that genetic factors play an indispensable role in the development of PCOS [5] and several

\footnotetext{
* Correspondence: hanzh80@yahoo.com

${ }^{\dagger}$ Equal contributors

${ }^{2}$ Center for Reproductive Medicine, Provincial Hospital Affiliated to Shandong University, Jinan, China

${ }^{3}$ National Research Center for Assisted Reproductive Technology and

Reproductive Genetics, Jinan, China

Full list of author information is available at the end of the article
}

candidate genes have been reported recently $[6,7]$. We performed the first GWAS for PCOS which followed by replication studies only for SNPs with p value less than $10 \mathrm{e}-6$, and finally identified three susceptibility loci (2p16.3, 2p21 and 9q33.3) [6]. However, other loci with $\mathrm{p}$ value around 10e-5 in GWAS, such as SNPs in gene USP34, remain intriguing and might also be potential risk factors of PCOS.

The USP34 gene is located on chromosome 2p15 and encodes a kind of deubiquitinating enzyme, which belongs to ubiquitin-specific protease family. Data obtained from COSMIC database [8] shows that somatic variations of USP34 are related to ovary tumor. Moreover, USP34 positively regulates Wnt signaling pathway [9], which plays an important role in gender differentiation, folliculogenesis, ovulation and other biological processes in reproduction [10]. The expression patterns of several genes in Wnt pathway are altered in PCOS (such as DKK1, a negative 
regulator of Wnt pathway) [11-13]. Taken together, it is assumed that USP34 may also have relationship with PCOS. As an extension of GWAS, here we conducted an independent case-control replication study to evaluate the association between USP34 and PCOS susceptibility.

\section{Methods}

This study was approved by Institutional Review Board for Reproductive Medicine of Shanghai Jiaotong University and Shandong University. A total of 1218 PCOS cases and 1057 unrelated controls were recruited consecutively from 2009 to 2013 at the Center for Reproductive Medicine, Renji Hospital, School of Medicine, Shanghai Jiaotong University and the Center for Reproductive Medicine, Provincial Hospital Affiliated to Shandong University. Among them, 94 PCOS cases, also born from Northern China, were collected at Renji Hospital from 2012 to 2013. Signed informed consent was obtained from each participant of this study.

PCOS diagnosis was based on the 2003 Rotterdam PCOS consensus criteria and other related diseases (such as congenital adrenal hyperplasia, Cushing's syndrome, androgen-secreting tumors, thyroid disease and hyperprolactinaemia) were excluded. In detail, PCOS can be diagnosed if at least two of the following three features are met: oligo- or anovulation, clinical and/or biochemical signs of hyperandrogenism and polycystic ovaries. Oligoor anovulation was referred to menstrual cycles of more than 35 days in length or a history of $\leq 8$ menstrual cycles in a year [1]; polycystic ovaries was defined as the presence of at least one ovary $>10 \mathrm{ml}$ or at least 12 follicles 2-9 $\mathrm{mm}$ in diameter by transvaginal ultrasound [4]. Hyperandrogenism was the presence of hirsutism (FerrimanGallwey score $\geq 6$ ) [14] or serum total testosterone $\geq 60 \mathrm{ng} / \mathrm{dl}$ [15]. The inclusion criteria for the control group were as follows: normal menstrual cycles, no hyperandrogenism and no polycystic ovaries (PCO) under ultrasound. All individuals who were taking medications such as oral contraceptives and metformin during last 3 months were excluded.

\section{Biochemical measurements}

Serum luteinizing hormone (LH) and testosterone (T) levels of subjects were measured by a chemiluminescent analyser (Beckman Access Health Company, Chaska, Minnesota, USA). The plasma glucose was measured by AU640 automatic biochemistry analyser (Olympus Company, Hamburg, Germany) and insulin was measured by chemiluminescent analyzer. Insulin resistance was calculated as fasting glucose $(\mathrm{mmol} / \mathrm{L})$ "fasting insulin $(\mathrm{mIU} / \mathrm{L}) / 22.5$ using homeostasis model assessment (HOMA-IR).

\section{SNP selection}

SNPs in USP34 were selected for replication study according to the following criteria: SNPs that exist in Affymetrix
Genome-Wide Human SNP Array 6.0 (Affymetrix, Santa Clara, CA, USA); can stand for a block; minor allele frequency (MAF) $>5 \%$ in Chinese Han population; statistically different $(P<10 \mathrm{e}-4)$ from our previous GWAS (see Additional file 1, Table S1); $\mathrm{r}^{2}$ of selected SNPs $<0.8$. Ultimately, rs17008097 and rs17008940 were selected to precede further replication study.

\section{SNP genotyping}

Genomic DNA was extracted from whole peripheral blood using QIAamp DNA mini kit (Qiagen, Hilden, Germany). Genotyping of SNPs was carried out by TaqMan-MGB probe assay (Invitrogen Trading, Shanghai, China), probes and primers were shown in Additional file 1, Table S2. Then, $5 \%$ of the samples were randomly selected for direct sequencing to validate the genotyping assays.

\section{Statistical analysis}

Numerical variables of clinical characteristics of PCOS cases and controls were expressed as mean \pm SD. HardyWeinberg equilibrium (HWE) and linkage disequilibrium (LD) tests were performed by Haploview software. The case-control genetic power was analyzed by Genetic Power Calculator [16]. A sample size $>721$ PCOS cases (case: control $=1)$ would provide $80 \%$ power $(\alpha=0.05)$, assuming higher risk allele frequency (A) of 0.05 and a genotype relative risk $(\mathrm{Aa})$ of 1.5. Frequencies of genotype and allele between PCOS subjects and controls were compared by Pearson Chi-square test, and the $\mathrm{p}$ value was adjusted by logistic regression to eliminate the effect of age and body mass index (BMI) using SPSS v.19.0 (SPSS Inc., Chicago, IL, USA). $P<0.05$ was considered statistically significant.

For genotype-phenotype analysis in PCOS patients, additive model $(+/+$ vs $+/-$ vs $-/-)$ was selected after comparison; one-way analysis of variance (ANOVA) test was used for phenotype comparison among different genotypes. Linear regression was used for age and BMI adjustment. Conservative Bonferroni test was used for multiple testing corrections.

\section{Results}

As shown in Table 1, the PCOS group was younger than the controls $(P<0.001)$. In addition, PCOS group had higher BMI, LH level and T level than the control group

Table 1 Characteristics of PCOS cases and controls

\begin{tabular}{llll}
\hline & PCOS & Control & p value \\
\hline Age (years) & $28.55 \pm 3.72$ & $31.84 \pm 4.74$ & $<0.001$ \\
BMI $\left(\mathrm{kg} / \mathrm{m}^{2}\right)$ & $25.12 \pm 4.35$ & $22.78 \pm 3.25$ & $<0.001$ \\
$\mathrm{LH}(\mathrm{IU} / \mathrm{L})$ & $10.03 \pm 5.93$ & $4.76 \pm 2.23$ & $<0.001$ \\
$\mathrm{~T}(\mathrm{ng} / \mathrm{dl})$ & $51.69 \pm 21.00$ & $42.35 \pm 18.46$ & $<0.001$
\end{tabular}

BMI: body mass index; LH: Luteinizing hormone; T: testosterone 
Table 2 Genotype and allele frequencies of USP34 in PCOS and controls

\begin{tabular}{|c|c|c|c|c|c|c|c|}
\hline & & & PCOS & Control & $P$ & $P_{\text {adjusted }}$ & OR/95 \% Cl \\
\hline \multirow[t]{5}{*}{ rs17008097 } & Genotype & $\mathrm{CC}$ & $548(45 \%)$ & $484(45.8 \%)$ & 0.807 & 0.791 & \\
\hline & & GC & $531(43.6 \%)$ & $447(42.3 \%)$ & & & \\
\hline & & GG & 139(11.4\%) & $126(11.9 \%)$ & & & \\
\hline & Allele & C & $1627(66.8 \%)$ & 1415(66.9 \%) & 0.918 & 0.791 & $0.993(0.878-1.124)$ \\
\hline & & G & 809(33.2 \%) & 699(33.1\%) & & & \\
\hline \multirow[t]{5}{*}{ rs17008940 } & Genotype & $\mathrm{CC}$ & $575(47.2 \%)$ & $505(47.8 \%)$ & 0.948 & 0.862 & \\
\hline & & $\mathrm{TC}$ & $513(42.1 \%)$ & $438(41.4 \%)$ & & & \\
\hline & & $\pi$ & 130(10.7\%) & $114(10.8 \%)$ & & & \\
\hline & Allele & $C$ & 1663(68.3\%) & 1448(68.5 \%) & 0.869 & 0.862 & $0.990(0.873-1.122)$ \\
\hline & & $\mathrm{T}$ & 773(31.7 \%) & $666(31.5 \%)$ & & & \\
\hline
\end{tabular}

$P$ adjusted: adjust the $\mathrm{p}$ value by age and $\mathrm{BMI}$; OR: Odd Ratio; $\mathrm{Cl}$ : Confidence Interval

$(P<0.001)$. In PCOS group, 90 patients present with hyperandrogenism and oligo-anovulation (HA + OA, 9.44\%), 10 patients present with hyperandrogenism and polycystic ovaries (HA + PCO, $1.05 \%), 620$ patients present with oligo-anovulation and polycystic ovaries (HA + PCO, $65.06 \%)$, and 233 patients present with full-phenotype (HA + OA + PCO, 24.45\%).

The genotype frequencies of the two polymorphisms in PCOS cases and controls were all in Hardy-Weinberg equilibrium $(P>0.05)$. Genotype and allele frequencies were summarized in Table 2 and no significant differences were observed between PCOS and controls. After age and BMI adjustment with logistic regression, no association was found. The minor allele frequencies (MAF) of the 2 SNPs in four subgroups of PCOS were further analyzed. No significant differences of MAF were observed between each subgroup of PCOS and controls in the present study (see Additional file 1, Table S3). Additionally, there was no statistical difference among three genetic models (additive, dominant and recessive) in genotype analysis (see Additional file 1, Table S4), thus additive model of genotype was selected for subsequent phenotype analysis.

In genotype-phenotype analysis, clinical and metabolic features were compared among different genotypes in PCOS subjects, rs17008940 was shown to be associated with BMI $(P=0.028)$ (Table 3$)$, however, the association was not significant after Bonferroni correction for multiple testing. The average level of BMI in TC and TT group was higher than that in $\mathrm{CC}$ group after age adjustment (TC vs CC $P=0.006, O R=1.042,95 \%$ CI 1.012-1.073; TT vs CC $P=0.037, O R=1.050,95 \%$ CI 1.003-1.100). But in control group, no significant difference was found in BMI among the three genotypes of $\operatorname{rs} 17008940 \quad(P=0.256)$. Additionally, there were no significant differences in LH, T or HOMA-IR among the PCOS cases carrying different genotypes of the two SNPs, even after age and BMI adjustment (Table 3, Table 4).

\section{Discussion}

As a powerful technique, GWAS shed new light on genetic study for complex diseases. GWAS data is obtained from computing and statistical analyses following SNP chips detection, so the results are bioinformatics rather than biological. However, GWAS itself owns some limitations and it is necessary to be validated through further replication studies. In general, p value of $5 * 10 \mathrm{e}-8$ was used as significant level for random variations in case-control GWAS with a power of $0.8[17,18]$. In our previous GWAS, only SNPs with p value $<10 \mathrm{e}-6$ were replicated to confirm the first step results [6]. However, some SNPs with $\mathrm{p}$ value around $10 \mathrm{e}-5$ were disputable. Recently we found two novel susceptibility genes YAP1 and LPP for PCOS from these SNPs $[7,19]$. So we selected two SNPs with $\mathrm{p}$ value around $10 \mathrm{e}-5$ in USP34 to validate whether USP34 was associated with PCOS. No association was

Table 3 Clinical and metabolic characteristics of PCOS cases in rs17008940 genotype subgroups

\begin{tabular}{|c|c|c|c|c|c|}
\hline Characteristics & $\mathrm{CC}(n=575)$ & $\mathrm{TC}(n=513)$ & $\Pi(n=130)$ & $P$ & $P_{\text {adjusted }}$ \\
\hline Age (years) & $28.83 \pm 3.73$ & $28.39 \pm 3.72$ & $28.02 \pm 3.66$ & 0.046 & - \\
\hline BMI $\left(\mathrm{kg} / \mathrm{m}^{2}\right)$ & $24.740 \pm 4.29$ & $25.418 \pm 4.34$ & $25.517 \pm 4.51$ & 0.028 & - \\
\hline $\mathrm{LH}(\mathrm{IU} / \mathrm{L})$ & $9.999 \pm 6.08$ & $10.028 \pm 5.88$ & $10.177 \pm 5.44$ & 0.957 & 0.500 \\
\hline $\mathrm{T}(\mathrm{ng} / \mathrm{dl})$ & $51.17 \pm 20.73$ & $51.74 \pm 21.41$ & $53.72 \pm 20.61$ & 0.485 & 0.505 \\
\hline HOMA-IR & $2.62 \pm 2.62$ & $2.70 \pm 2.12$ & $2.79 \pm 2.32$ & 0.653 & 0.399 \\
\hline
\end{tabular}

HOMA-IR: homeostasis model assessment; $P$ adjusted: adjust the $\mathrm{p}$ value by age and $\mathrm{BMI}$ in logistic regression 
Table 4 Clinical and metabolic characteristics of PCOS cases in rs17008097 genotype subgroups

\begin{tabular}{|c|c|c|c|c|c|}
\hline Characteristics & $C C(n=548)$ & $\mathrm{GC}(n=531)$ & $\mathrm{GG}(n=139)$ & $P$ & $P_{\text {adjusted }}$ \\
\hline Age(years) & $28.82 \pm 3.79$ & $28.44 \pm 3.64$ & $27.97 \pm 3.73$ & 0.044 & - \\
\hline $\mathrm{BMI}\left(\mathrm{kg} / \mathrm{m}^{2}\right)$ & $24.824 \pm 4.26$ & $25.287 \pm 4.37$ & $25.581 \pm 4.51$ & 0.106 & - \\
\hline LH (IU/L) & $10.000 \pm 6.12$ & $9.963 \pm 5.81$ & $10.399 \pm 5.61$ & 0.746 & 0.393 \\
\hline $\mathrm{T}(\mathrm{ng} / \mathrm{dl})$ & $51.446 \pm 20.14$ & $51.897 \pm 21.97$ & $51.868 \pm 20.69$ & 0.940 & 0.904 \\
\hline HOMA-IR & $2.642 \pm 2.61$ & $2.676 \pm 2.19$ & $2.773 \pm 2.24$ & 0.800 & 0.415 \\
\hline
\end{tabular}

HOMA-IR: homeostasis model assessment; $P$ adjusted: adjust the $\mathrm{p}$ value by age and BMI in logistic regression

replicated in the present study. Therefore, SNPs with $\mathrm{p}$ value around $10 \mathrm{e}-5$ in GWAS were controvertible and demanded for independent and large cohort of samples for verification.

Although rs17008940 was not shown to be related to PCOS, it might confer slight risk to the elevated BMI in PCOS. However, this slightly association possibly results from a selection bias derived from the patients and controls being recruited in an infertility clinical center and not from the general population. Hence, the results need further validation. Higher BMI was one of the important characteristics in PCOS and over 50 \% PCOS women were overweight or obesity [20,21]. Consistent with our results, previous studies also showed FTO and MC4R were associated with increased BMI in PCOS subjects rather than PCOS itself [22]. Abundant evidence have linked Wnt signals to the regulation of adipogenesis $[23,24]$ and body fat distribution [25]. For example, Christodoulides et al. reported that mutation $\mathrm{C} 256 \mathrm{Y}$ in WNT10B was associated with overweight or obesity because the mutation was unable to activate canonical Wnt pathway [26]; and Choi et al. found that indirubin-3'-oxime (I3O), also an activator of the Wnt signaling like USP34, inhibited the development of obesity in high-fat diet fed mice [27]. Moreover, besides acting as an activator of Wnt pathway, USP34 was also found to play a role in $N F k B$ signal regulation in T lymphocytes [28] and DNA damage response control as it was the downstream of ATM/ATR checkpoint kinase $[29,30]$. USP34 may indirectly participate in the pathophysiology of PCOS by elevating BMI, but further studies were still needed to evaluate the function of USP34 in the BMI increase among PCOS women.

Some limitations of the present replication study should be mentioned. First, the sample size of this replication study was relatively small (rs17008940, OR = 1.010; rs17008097, $O R=1.007$ ) and this replication study maybe not sufficient to detect the potential association between USP34 gene and PCOS. Second, only 2 SNPs were chosen which may cause incomplete coverage of the gene variations. Third, the recruited subjects were all Han Chinese women and the result could not represent other population.

\section{Conclusions}

In conclusion, the present study found that polymorphisms of USP34 gene may not be associated with PCOS women among Han Chinese population. SNPs with $p$ value around $10 \mathrm{e}-4 \sim 10 \mathrm{e}-6$ in GWAS were disputable and requiring replication studies for validation. Large welldesigned and population-based studies are warranted to confirm our findings.

\section{Additional file}

Additional file 1: Table S1. GWAS database of USP34. Table S2. Probes and primers of the three SNPs. Table S3. Allele frequencies comparison of USP34 in four subgroups of PCOS and controls. Table S4. Genotype frequencies comparison in PCOS and controls using different genetic models.

\section{Competing interests}

The authors declare that they have no competing interests.

\section{Authors' contributions}

SZ, YT and WZ carried out studies, and drafted the manuscript. YN carried out the biochemical measurements. YT, XX and TL participated in the design of the study and performed the statistical analysis. $\mathrm{HL}$ and $\mathrm{TH}$ helped to select subjects. $\mathrm{HZ}$ and ZJC conceived of the study, and participated in its design and coordination and helped to draft the manuscript. All authors read and approved the final manuscript.

\section{Acknowledgements}

The authors thank Li You, Jiangtao Zhang, Guangyu Li and Changming Zhang for technical support and sample collection. This work was supported by the National Basic Research Program of China (973 program)

(2012CB944700, 2011CB944502), the National Natural Science Foundation of China $(31371453,81430029)$, the Scientific Research Foundation of Shandong Province of Outstanding Young Scientist (2012BSE27089) and the Doctoral Innovation Fund Projects from Shanghai Jiaotong University School of Medicine (Grant No. BXJ201324).

\section{Author details}

${ }^{1}$ Shanghai Key Laboratory for Assisted Reproduction and Reproductive Genetics, Center for Reproductive Medicine, Renji Hospital, School of Medicine, Shanghai Jiaotong University, Shanghai 200135, China. ${ }^{2}$ Center for Reproductive Medicine, Provincial Hospital Affiliated to Shandong University, Jinan, China. ${ }^{3}$ National Research Center for Assisted Reproductive Technology and Reproductive Genetics, Jinan, China. ${ }^{4}$ The Key laboratory for Reproductive Endocrinology of Ministry of Education, Jinan, China.

${ }^{5}$ Shandong Provincial Key Laboratory of Reproductive Medicine, Jinan 250021, China. ${ }^{6}$ Department of joint and bone oncology, Provincial Hospital Affiliated to Shandong University, Jinan 250021, China.

Received: 3 March 2015 Accepted: 8 May 2015

Published online: 15 May 2015 


\section{References}

1. Azziz R, Woods KS, Reyna R, Key TJ, Knochenhauer ES, Yildiz BO. The prevalence and features of the polycystic ovary syndrome in an unselected population. J Clin Endocrinol Metab. 2004;89(6):2745-9.

2. Goodarzi MO, Azziz R. Diagnosis, epidemiology, and genetics of the polycystic ovary syndrome. Best Pract Res Clin Endocrinol Metab. 2006;20(2):193-205.

3. Li R, Zhang Q, Yang D, Li S, Lu S, Wu X, et al. Prevalence of polycystic ovary syndrome in women in China: a large community-based study. Hum. Reprod. 2013;28(9):2562-9.

4. Rotterdam EA-SPCWG. Revised 2003 consensus on diagnostic criteria and long-term health risks related to polycystic ovary syndrome. Fertil Steril. 2004;81(1):19-25.

5. Calogero AE, Calabro V, Catanuso M, Condorelli RA, La Vignera S. Understanding polycystic ovarian syndrome pathogenesis: an updated of its genetic aspects. J Endocrinol Invest. 2011;34(8):630-44.

6. Chen ZJ, Zhao H, He L, Shi Y, Qin Y, Shi Y, et al. Genome-wide association study identifies susceptibility loci for polycystic ovary syndrome on chromosome 2p16.3, 2p21 and 9q33.3. Nat Genet. 2011;43(1):55-9.

7. Li T, Zhao H, Zhao X, Zhang B, Cui L, Shi Y, et al. Identification of YAP1 as a novel susceptibility gene for polycystic ovary syndrome. J Med Genet. 2012:49(4):254-7

8. COSMIC database. Wellcome Trust Sanger Institute, England. 2004. http:// cancer.sanger.ac.uk/cancergenome/projects/cosmic. Accessed 4 Feb 2004.

9. Lui T, Lacroix C, Ahmed SM, Goldenberg SJ, Leach CA, Daulat AM, et al. The ubiquitin-specific protease USP34 regulates axin stability and Wnt/ beta-catenin signaling. Mol Cell Biol. 2011;31(10):2053-65.

10. Richards JS, Russell DL, Ochsner S, Hsieh M, Doyle KH, Falender AE, et al. Novel signaling pathways that control ovarian follicular development, ovulation, and luteinization. Recent Prog Horm Res. 2002;57:195-220.

11. Corton M, Botella-Carretero Il, Benguria A, Villuendas G, Zaballos A, San Millan JL, et al. Differential gene expression profile in omental adipose tissue in women with polycystic ovary syndrome. J Clin Endocrinol Metab. 2007;92(1):328-37.

12. Jansen E, Laven JS, Dommerholt HB, Polman J, van Rijt C, van den Hurk C, et al. Abnormal gene expression profiles in human ovaries from polycystic ovary syndrome patients. Mol Endocrinol. 2004;18(12):3050-63.

13. Kenigsberg S, Bentov Y, Chalifa-Caspi V, Potashnik G, Ofir R, Birk OS. Gene expression microarray profiles of cumulus cells in lean and overweight-obese polycystic ovary syndrome patients. Mol Hum Reprod. 2009;15(2):89-103.

14. Ferriman D, Gallwey JD. Clinical assessment of body hair growth in women. J Clin Endocrinol Metab. 1961;21:1440-7

15. Shi Y, Gao X, Sun X, Zhang P, Chen Z. Clinical and metabolic characteristics of polycystic ovary syndrome without polycystic ovary: a pilot study on Chinese women. Fertil Steril. 2008;90(4):1139-43.

16. Purcell S, Cherny SS, Sham PC. Genetic Power Calculator: design of linkage and association genetic mapping studies of complex traits. Bioinformatics. 2003;19(1):149-50.

17. Newton-Cheh C, Hirschhorn JN. Genetic association studies of complex traits: design and analysis issues. Mutat Res. 2005;573(1-2):54-69.

18. Lewis CM. Genetic association studies: design, analysis and interpretation. Brief Bioinform. 2002;3(2):146-53.

19. Zhang B, Zhao H, Li T, Gao X, Gao Q, Tang R, et al. Association study of gene LPP in women with polycystic ovary syndrome. PLoS One. 2012;7(10), e46370.

20. Legro RS, Kunselman AR, Dodson WC, Dunaif A. Prevalence and predictors of risk for type 2 diabetes mellitus and impaired glucose tolerance in polycystic ovary syndrome: a prospective, controlled study in 254 affected women. J Clin Endocrinol Metab. 1999:84(1):165-9.

21. Shi Y, Guo M, Yan J, Sun W, Zhang X, Geng L, et al. Analysis of clinical characteristics in large-scale Chinese women with polycystic ovary syndrome. Neuro Endocrinol Lett. 2007;28(6):807-10.

22. Ewens KG, Jones MR, Ankener W, Stewart DR, Urbanek M, Dunaif A, et al. FTO and MC4R gene variants are associated with obesity in polycystic ovary syndrome. PLoS One. 2011;6(1), e16390.

23. Ross SE, Hemati N, Longo KA, Bennett CN, Lucas PC, Erickson RL, et al. Inhibition of adipogenesis by Wnt signaling. Science. 2000;289(5481):950-3.

24. Bennett CN, Ross SE, Longo KA, Bajnok L, Hemati N, Johnson KW, et al. Regulation of Wnt signaling during adipogenesis. J Biol Chem. 2002;277(34):30998-1004.

25. Gesta S, Bluher M, Yamamoto Y, Norris AW, Berndt J, Kralisch S, et al. Evidence for a role of developmental genes in the origin of obesity and body fat distribution. Proc Natl Acad Sci U S A. 2006;103(17):6676-81.
26. Christodoulides C, Scarda A, Granzotto M, Milan G, Dalla Nora E, Keogh J, et al. WNT10B mutations in human obesity. Diabetologia. 2006;49(4):678-84.

27. Choi OM, Cho YH, Choi S, Lee SH, Wha Seo S, Kim HY, et al. The small molecule indirubin-3'-oxime activates Wnt/beta-catenin signaling and inhibits adipocyte differentiation and obesity. Int J Obes. 2013;38(8):1044-52.

28. Poalas K, Hatchi EM, Cordeiro N, Dubois SM, Leclair HM, Leveau C, et al. Negative regulation of NF-kappaB signaling in T lymphocytes by the ubiquitin-specific protease USP34. Cell Commun Signal. 2013;11(1):25.

29. Mu JJ, Wang $Y$, Luo H, Leng M, Zhang J, Yang T, et al. A proteomic analysis of ataxia telangiectasia-mutated (ATM)/ATM-Rad3-related (ATR) substrates identifies the ubiquitin-proteasome system as a regulator for DNA damage checkpoints. J Biol Chem. 2007;282(24):17330-4.

30. Sy SM, Jiang J, O WS, Deng Y, Huen MS. The ubiquitin specific protease USP34 promotes ubiquitin signaling at DNA double-strand breaks. Nucleic Acids Res. 2013:41(18):8572-80.

\section{Submit your next manuscript to BioMed Central and take full advantage of:}

- Convenient online submission

- Thorough peer review

- No space constraints or color figure charges

- Immediate publication on acceptance

- Inclusion in PubMed, CAS, Scopus and Google Scholar

- Research which is freely available for redistribution 